\title{
AVALIAÇÃO DA INTENSIDADE DE ESFORÇO DURANTE AULAS DE GRUPO: UMA COMPARAÇÃO ENTRE AS MODALIDADES DE STEP COREOGRAFADO E STEP ATLÉTICO
}

EVALUATION OF THE EFFORT INTENSITY DURING GROUP CLASSES: A COMPARISON BETWEEN THE MODALITIES OF CHOREOGRAPHED STEP AND ATHLETIC STEP

EVALUACIÓN DE LA INTENSIDAD DE ESFUERZO DURANTE LAS CLASES DE GRUPO: UNA COMPARACIÓN ENTRE LAS MODALIDADES DE STEP COREOGRAFADO Y STEP ATLÉTICO.

\author{
Ana Simão (anasimao93@hotmail.com)* \\ Claudia Vaz (claudiasvaz92@gmail.com)** \\ Bernardete Jorge (bernardete@ipg.pt)*** \\ Carolina Vila-Chã (cvilacha@ipg.pt)
}

\section{RESUMO}

Este estudo teve como objetivo avaliar e comparar a intensidade de esforço em duas aulas de step [coreografado (SC) e atlético (SA)]. As 8 participantes da amostra (género feminino, 21,3 $\pm 1,1$ anos) foram submetidas a duas sessões de 45 minutos, com o step a 15cm de altura e cadência musical de 132bpm, com um intervalo de 48h entre sessões. A intensidade de esforço foi avaliada através da monitorização da frequência cardíaca (FC). Foi calculada a FC reserva e determinadas as zonas de intensidade (ACSM, 2011). Foi ainda avaliada a perceção subjetiva de esforço (PSE - escala de Borg). A FC média nas aulas

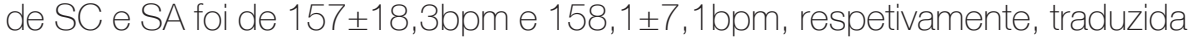

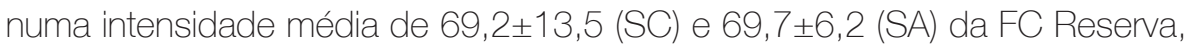
sem diferenças significativas entre as duas vertentes de step ( $p>0,73)$. Contudo, na aula de SA as participantes passaram 70 $\pm 8,8 \%(p>0,05)$ do tempo em atividade vigorosa enquanto no SC passaram 50 $\pm 10,3 \%$ (p>0,05). Não foram encontradas diferenças significativas relativamente ao tempo despendido noutras zonas de treino, entre modalidades. Na aula de SA (16,67 \pm

1), a PSE foi significativamente superior à aula de SC $(15,44 \pm 0,73)$. Ambas as modalidades permitem praticar exercício físico de intensidade moderada a 
vigorosa, concordando com as recomendações do ACSM (2011).

Palavras-chave: Step; Frequência cardíaca; Perceção subjetiva de esforço.

\section{ABSTRACT}

The purpose of this study was to evaluate and compare the effort intensity during two step classes [choreographed (CS) and athletic (AS)]. The 8 participants (female, $21.3 \pm 1.1$ years) were submitted to two 45 minute sessions, with the step at $15 \mathrm{~cm}$ and musical cadence of 132bpm, 48h interval between sessions. Effort intensity was assessed by heart rate $(H R)$ monitoring. The HR reserve was calculated and the zones of intensity determined (ACSM, 2011). The Rated Perceived Exertion (PSE - Borg scale) was also evaluated. The mean HR in CS and AS classes was $157 \pm 18.3 \mathrm{bpm}$ and $158.1 \pm 7.1 \mathrm{bpm}$, respectively, translated into a mean intensity of $69.2 \pm 13.5$ (CS) and $69.7 \pm 6.2$ (AS) of HR reserve, without significant differences between the two step modalities (p>0.73). However, in AS class, the participants spent $70 \pm$ $8.8 \%$ ( $p>0.05$ ). of the time in vigorous activity, while in CS they passed $50 \pm 10.3 \%$ ( $p>0.05$ ). No significant differences were found regarding the time spent in other training zones, between modalities. In AS class (16.67 \pm 1$)$, the PSE was significantly higher than in CS class (15.44 \pm 0.73). Both modalities allow to practice moderate to vigorous physical exercise, in agreement with the ACSM's (2011) recommendations.

Keywords: Step; Heart rate; Perceived Exertion Scale.

\section{RESUMEN}

Este estudio tuvo como objetivo evaluar y comparar la intensidad de esfuerzo en dos clases de step [coreografiado (SC) y atlético (SA)]. Las 8 participantes de la muestra (género femenino, 21,3 \pm 1,1 años) fueron sometidas a dos sesiones de 45 minutos, con el step a $15 \mathrm{~cm}$ y cadencia musical de 132bpm, intervalo de 48h entre sesiones. La intensidad de esfuerzo se evaluó mediante la monitorización de la frecuencia cardíaca (FC). Se calculó la FC Reserva y se determinaron las zonas de intensidad (ACSM, 2011). Se evaluó la percepción subjetiva del esfuer- 
zo (PSE - escala de Borg). La FC media en las clases de SC y SA fue de $157 \pm 18,3 \mathrm{bpm}$ y 158,1 \pm 7,1 bpm, respectivamente, traducida en una intensidad media de 69,2 $\pm 13,5$ (SC) y 69,7 \pm 6,2 (SA) de la FC reserva, sin diferencias significativas entre las dos variantes de step ( $\mathrm{p}$ > $0,73)$. Sin embargo, en la clase de SA las participantes pasaron $70 \pm$ 8,8\% ( $p>0.05$ ) del tiempo en actividad vigorosa mientras en el SC pasaron $50 \pm 10,3 \%(p>0,05)$. No se encontraron diferencias significativas con respecto al tiempo empleado en otras zonas de entrenamiento, entre modalidades. En la clase de SA (16,67 \pm 1 ), la PSE fue significativamente superior a la clase de SC $(15,44 \pm 0,73)$. Ambas modalidades permiten practicar ejercicio físico de intensidad moderada a vigorosa, concordando con las recomendaciones del ACSM (2011).

\footnotetext{
Palabras clave: Step; Frecuencia cardíaca; Escala de Esfuerzo Percibido.
}

* Mestre em Ciências do Desporto, especialidade Desportos de Academia e licenciada em Desporto pelo Instituto Politécnico da Guarda, licenciada em Desporto pela mesma instituição

** Estudante de Mestrado em Ciências do Desporto, especialidade Desportos de Academia, do Instituto Politécnico da Guarda. Licenciada em Desporto pela mesma instituição.

*** Professora Adjunta na Escola Superior de Educação, Comunicação e Desporto do Instituto Politécnico da Guarda, Portugal. Membro da Unidade de Investigação para o Desenvolvimento do Interior (UDI). Especialista em Ciências do Desporto, Atividades de Academia, pelo Instituto Politécnico do Porto.

**** Professora Adjunta na Escola Superior de Educação, Comunicação e Desporto do Instituto Politécnico da Guarda, Portugal. Membro efetivo do Centro de Investigação em Desporto, Saúde e Desenvolvimento (CIDESD). Licenciada e mestre em Ciências do Desporto pela Faculdade de Desporto da Universidade do Porto (UP) Doutorada em Engenharia Biomédica pela Faculdade de Engenharia da UP. 


\section{INTRODUÇÃO}

Nas últimas décadas tem sido demonstrado que a prática regular de atividade física e bons hábitos alimentares são fundamentais para a gestão eficaz do peso corporal e redução do risco de desenvolvimento de doenças cardiovasculares, diabetes tipo II e alguns tipos de cancro (Reiner, Niermann, Jekauc, \& Woll, 2013). De acordo com o American College of Sport Medicine ( ACSM, 2011), a redução significativa do risco de todas as causas de mortalidade precoce pode ser conseguida se o indivíduo alcançar um dispêndio energético semanal mínimo de 1000kcal com atividade física. Para este efeito o ACSM, recomenda a prática de exercício cardiorrespiratório, no mínimo cinco vezes por semana, com duração mínima de 150 minutos semanais, a uma intensidade moderada ou, em alternativa, um mínimo de 3 vezes por semana, com duração mínima de 75 minutos semanais, a uma intensidade vigorosa (ACSM, 2011).

As aulas de exercício físico em grupo são privilegiadas por muitos indivíduos, em particular por mulheres, como uma forma de manterem a sua prática regular de atividade física (DeSimone, 2012). Atualmente, este tipo de aulas têm uma grande expressão na área do fitness e de entre as inúmeras modalidades disponíveis, o step continua a ser uma das aulas mais populares na maior parte do ginásios (Arslan, 2011). Esta modalidade é caracterizada por uma combinação de diferentes gestos técnicos de membros inferiores e superiores com subidas e descidas sucessivas da plataforma de step, que são estruturados em blocos coreográficos, repetidos várias vezes ao longo da aula ao ritmo de música com características específicas (La Torre et al., 2005). De acordo com Jaywant (2005), o step pode ser considerado um modo de exercício físico eficaz para a melhoria da função cardiorrespiratória e diminuição de massa gorda, em particular entre mulheres pouco ativas. Vários estudos indicam também que as respostas fisiológicas agudas associadas à pratica destas modalidades, quando conjugadas com a frequência semanal aconselhada, podem permitir o alcance das recomendações emanadas pelo ACSM. Ou seja, a prática regular, com duração mínima de 150 minutos semanais, 5 vezes por semana, a uma intensidade moderada ou, em alternativa, um mínimo de três vezes por semana, com duração mínima de 75 minutos semanais, a uma intensi- 
dade vigorosa, são suficientes para obter efeitos benéficos da atividade física sobre a saúde dos indivíduos (De Angelis, Vinciguerra, Gasbarri, \& Pacitti, 1998; Laukkanen et al., 2001). No entanto, as respostas fisiológicas alcançadas durante uma aula de step, podem ser variáveis dependendo das metodologias seguidas pelo instrutor.

As vertentes comercias mais populares das aulas step seguem duas tendências metodológicas distintas: uma mais centrada na sua vertente coreografada, em que as habilidades motoras são realizadas em sintonia com um determinado estilo musical e que tipicamente acarretam uma maior complexidade de execução técnica (step coreografado) e; outra mais centrada no seu impacto fisiológico, constituída essencialmente por habilidades motoras básicas repetidas ao longo de vários blocos (step atlético). A manipulação de parâmetros como a cadência musical, a alteração da altura do step, ou seleção de ações motoras que englobem maior ou menor quantidade de massa muscular, pode promover diferentes respostas agudas fisiológicas (Martinovic \& Novaes, 2002). Contudo, e apesar das boas taxas de adesão a este tipo de aulas, não existem dados que descrevam e/ou comparem as respostas fisiológicas associadas a estas variantes do step, nem que identifiquem em que medida estas permitem cumprir as recomendações de atividade física acima mencionadas. Assim, este estudo teve como objetivo estudar e comparar a intensidade de esforço exigida pela prática de aula de step coreografado vs. step atlético. Para este efeito foi avaliada a frequência cardíaca (FC) e a perceção subjetiva de esforço (PSE).

\section{METODOLOGIA}

\section{AMOSTRA}

A amostra foi constituída por 8 elementos do género feminino, praticantes das aulas de grupo, com idades compreendidas entre os 20 e os 23 anos. Todas as participantes foram informadas dos procedimentos do estudo e deram o seu consentimento informado. A Tabela 1 apresenta a caracterização da amostra. 
Tabela 1 - Média e desvio-padrão (DP) das variáveis idade, massa corporal, altura, índice de massa corporal (IMC) e frequência cardíaca (FC) de repouso da amostra.

\begin{tabular}{|c|c|c|c|c|c|}
\hline & Idade & $\begin{array}{c}\text { Massa } \\
\text { corporal } \\
(\mathrm{kg})\end{array}$ & Altura & IMC & $\begin{array}{c}\text { FC Repou- } \\
\text { so (bpm) }\end{array}$ \\
\hline $\begin{array}{c}\text { Média } \pm \\
\text { DP }\end{array}$ & $\begin{array}{c}21,3 \pm \\
1,12\end{array}$ & $\begin{array}{c}58,8 \pm \\
7,89\end{array}$ & $\begin{array}{c}1,65 \pm \\
0,06\end{array}$ & $\begin{array}{c}21,6 \pm \\
2,68\end{array}$ & $\begin{array}{c}63,9 \pm \\
7,79\end{array}$ \\
\hline
\end{tabular}

\section{PROCEDIMENTOS EXPERIMENTAIS}

A recolha de dados deste estudo foi realizada durante três sessões independentes. A primeira sessão correspondeu ao esclarecimento do objetivo e procedimentos do estudo e às medições da massa corporal e altura. Nesta, foi solicitado à amostra que medisse a sua FC de repouso ao acordar, de forma a ter-se um valor mais fiável. As participantes, embora já familiarizadas com a forma de aceder à FC de repouso, foram novamente instruídas sobre a forma de avaliar corretamente parâmetro fisiológico. Na segunda e terceira sessões as participantes realizaram as aulas de grupo (step coreografado e step atlético, respetivamente). Durante estas sessões procedeu-se à avaliação da intensidade do esforço das aulas com base na FC e PSE. Entre a segunda e a terceira sessão houve um intervalo de 24 horas, sendo estas realizadas à mesma hora do dia (12h30-13h30). Foi solicitado às participantes que entre sessões de avaliação a intensidade da sua atividade física diária fosse mantida em níveis leves ou muito leves.

Cada aula de grupo foi lecionada como uma prática normal, isto é sem o reconhecimento prévio da coreografia por parte das praticantes, com a duração total de aproximadamente 57 minutos, em que os 7 minutos iniciais foram de aquecimento e os 5 minutos finais de retorno à calma. Ambas as aulas foram lecionadas com uma cadência musical de 132 bpm, blocos de 32 tempos, plataforma no nível 1 (15 cm) e com o método de ensino de adição. A FC foi avaliada durante as duas sessões de step e a PSE foi avaliada no final de cada sessão. 


\section{RECOLHA E TRATAMENTO DOS DADOS}

\section{FREQUÊNCIA CARDÍACA E ACELEROMETRIA}

Na segunda e terceira sessões, as participantes usaram uma banda com sensores de frequência cardíaca (Zephyr ${ }^{\text {TM }}$ BioHarness ${ }^{\text {TM }}$ 3). Este dispositivo, permite registar a FC através de sensores que detetam formas de onda R, correspondendo cada onda a um batimento por minuto (bpm). A frequência de amostragem deste sinal foi de $250 \mathrm{~Hz}$. Os dados recolhidos durante cada uma das sessões foram transmitidos telemetricamente para um portátil com o software de aquisição instalado (Omnisense 3.7.15). Posteriormente, os dados foram exportados para ficheiros formato Microsoft@ Excel e tratados através de uma rotina Matlab construída para:

- Calcular a FC de Reserva (FCreserva) de cada participante com base na fórmula de Karvonen (Karvonen, Kentala, \& Musta, 1957);

- A FC máxima (FCmáx) foi estimada com base na fórmula de Fox (Fox, Naughton, \& Haskell, 1971);

- Calcular o tempo total de aula despendido em diferentes intervalos de intensidade de esforço indicados pelo ACSM (muito leve, leve, moderado, vigoroso,) com base na FCreserva e na FCmáx;

- Determinar a percentagem de tempo total passado em cada um dos níveis de intensidade em função das variáveis FCreserva e FCmáx. O valor é expresso em função da duração total da parte fundamental da aula (aproximadamente 45 minutos).

\section{PERCEÇÃO SUBJETIVA DE ESFORÇO}

A avaliação da PSE foi efetuada através da escala de Borg de 6 a 20 (Borg, 1982). As participantes foram familiarizadas com a escala de Borg, tendo-lhes sido informado que, na escala de 6 a 20, o valor 6 
representa um esforço muito fácil e 20 corresponde a um esforço extenuante. Os valores situados neste intervalo representam a progressão do esforço entre muito fácil e extenuante. No final de cada sessão, foi-Ihes pedido para indicar a sua perceção de esforço com base nesta escala.

\section{ANÁLISE ESTATÍSTICA}

A análise estatística dos dados compreendeu dois blocos. Num primeiro bloco, realizou-se a análise exploratória e descritiva dos dados. Num segundo bloco, efetuou-se a análise inferencial dos mesmos. No que diz respeito à análise exploratória e descritiva, foram utilizados medidas de tendência central (média) e de dispersão (desvio-padrão e coeficiente de variação). Foi também realizada a análise da simetria (Skewness) e de achatamento (Kurtosis) das curvas, bem como da normalidade da distribuição através do teste de Kolmogorov-Smirnov. Dado que a distribuição da amostra não cumpre o pressuposto da normalidade, a análise inferencial (para comparação dos parâmetros baseados na frequência cardíaca e perceção subjetiva de esforço) foi efetuada através de um teste não paramétrico para medidas repetidas (teste de Wilcoxon) (Zar, 2010).

\section{RESULTADOS}

Na Tabela 2 estão representados os valores médios da PSE, da FC e das percentagens da FCmáx e de FCreserva obtidos durante a parte fundamental da sessão de ambas as modalidades avaliadas.

Tabela 2 - Valores médios e desvio padrão da percepção subjetiva do esforço (PSE), frequência cardíaca (FC), percentagem da frequência cardíaca máxima (\% FCmáx) e percentagem da frequência cardíaca máxima (\% FCreserva) da aula de step coreografado e step atlético.

\begin{tabular}{|l|c|c|}
\hline Variáveis & Step & Step Atlético \\
\hline PSE & $15,44 \pm 0,73$ & $16,67 \pm 1^{*}$ \\
\hline FC & $157,1 \pm 18,3$ & $158,1 \pm 7,1$ \\
\hline \%FC máx & $79 \pm 9,2$ & $79,5 \pm 3,3$ \\
\hline \%FC Reserva & $69,2 \pm 13,5$ & $69,7 \pm 6,2$ \\
\hline
\end{tabular}

$* p<0,05$ 
Não foram verificadas diferenças significativas no que diz respeito ao valor médio da frequência cardíaca, à \% de FCmáx e à \% de FCreserva. Contudo, relativamente à perceção subjetiva de esforço, podemos verificar que as participantes identificaram a aula de step atlético como exigindo um maior esforço quando comparada com a aula de step coreografado (tabela 2).

A Tabela 3 apresenta os valores médios do intervalo de tempo passado em cada nível de intensidade, para cada uma das aulas de step. Com base nos resultados desta tabela, podemos verificar que na aula de step atlético as participantes, estiveram, em média, mais tempo numa intensidade vigorosa do que na aula de step coreografado, independentemente da variável analisada ( \% FCreserva ou da \% FCmáx).

Tabela 3 - Valor médio e respetivo desvio-padrão do intervalo de tempo em minutos (min) despendido em cada intervalo de intensidade, para cada uma das modalidades de step. Os níveis de intensidade foram definidos com base nos parâmetros de percentagem de frequência cardíaca máxima ( $\%$ F Cmáx) e frequência cardíaca de reserva (\% FCreserva).

\begin{tabular}{|c|c|c|c|}
\hline \multirow{2}{*}{ Variáveis } & \multirow{2}{*}{ Níveis de Intensidade } & \multicolumn{2}{|c|}{ Tempo (min) } \\
\hline & & Step Coreografado & Step Atlético \\
\hline \multirow[t]{5}{*}{$\%$ FCreserva } & Muito Leve $(<30)$ & $1,9 \pm 1,7^{\dagger}$ & $0,3 \pm 0,5^{\dagger}$ \\
\hline & Leve (30-39) & $3,8 \pm 3,6$ & $1,1 \pm 1,4^{\dagger}$ \\
\hline & Moderado (40-59) & $9,0 \pm 4$ & $9,4 \pm 4,0$ \\
\hline & Vigoroso (60-89) & $23,5 \pm 8,8$ & $32,5 \pm 4,5^{* \dagger}$ \\
\hline & Quase Máxima ( $\geq 90)$ & $5,1 \pm 6,4^{\dagger}$ & $2,7 \pm 3,4$ \\
\hline \multirow[t]{5}{*}{ \% FC máx } & Muito Leve $(<57)$ & $5,0 \pm 5,1$ & $0,7 \pm 0,7$ \\
\hline & Leve (57-63) & $3,4 \pm 3,6$ & $3,0 \pm 1,9$ \\
\hline & Moderado (64-76) & $11,3 \pm 4,6$ & $12,7 \pm 4,1$ \\
\hline & Vigoroso (77-95) & $21,1 \pm 6,5$ & $29,9 \pm 5,9 *$ \\
\hline & Quase Máxima ( $\geq 96)$ & $2,4 \pm 4,2$ & $0,5 \pm 0,8$ \\
\hline
\end{tabular}

* - tempo em intensidade vigorosa é significativamente superior na modalidade de step atlético, independentemente do parâmetro de intensidade utilizado para avaliar o esforço $(p>0,05) ; \dagger$ - quantidade de tempo passado em cada intervalo de intensidade difere significativamente em função do parâmetro fisiológico selecionado $(p>0,05)$.

Dependendo do parâmetro fisiológico analisado, as participantes passam em média 64 a 71\% do tempo da aula em intensidades vigorosas (FCmáx e FCreserva, respetivamente). No step coreografado o intervalo de tempo em intensidade quase máxima é superior, embora 
não seja estatisticamente significativo. $\bigcirc$ valor médio foi influenciado pelo resultado de 2 participantes que passaram aproximadamente 13 minutos neste regime de intensidade durante a parte fundamental da aula. Relativamente ao intervalo de tempo em regime de intensidade moderada, os resultados foram semelhantes para ambas as aulas independentemente do tipo de parâmetro fisiológico analisado. Quando analisado em simultâneo o intervalo de tempo passado em regimes de intensidade muito leve ou leve, verifica-se que, em média as participantes despendem mais tempo nestes regimes durante a aula de step coreografado do que durante a aula de step atlético. Contudo, estes resultados não são estatisticamente significativos.

De acordo com os resultados apresentados na Tabela 3, o tipo de parâmetro fisiológico selecionado afeta significativamente a quantificação do tempo passado em cada regime de intensidade. Acrescesse o facto destes não afetarem de igual forma os diferentes intervalos de intensidade para cada uma das modalidades de step.

\section{DISCUSSÃO}

Os resultados deste estudo indicam que, durante a parte fundamental de ambas as modalidades de step, é possível alcançar os níveis de intensidade recomendados pelo ACSM para adultos saudáveis (ACSM, 2011). De acordo com perceção subjetiva de esforço das participantes, ambas as vertentes da aula de step foram classificadas como sendo de intensidade vigorosa (PSE entre 14 e 17, ACSM, 2011). Este parâmetro subjetivo de avaliação foi confirmado pela avaliação de parâmetros fisiológicos (\%FCmáx e \%FCreserva). Nas aulas de step coreografado e step atlético foram registadas valores de \% FCmáx (79\% e 79,5\%) e da \% FCreserva (69,2\% e 69,2\%) que, de acordo com o ACSM (2011), também se classificam num nível de intensidade vigorosa (\% FCmáx 77-95 e \% FC reserva 60-89). Contudo, estes resultados apenas revelam o comportamento médio ao longo de toda aula, podendo mascarar intervalos de tempo de aula passados noutros regimes de intensidade. A análise contínua das variáveis fisiológicas, permitiu verificar que uma parte significativa da aulas de step coreografado e step atlético é passada em regime de intensidade vigorosa [45\% a 50\% e 64 a 70\% do tempo 
da parte fundamental, dependendo do parâmetro fisiológico analisado (\%FCmáx vs. \% FC reserva)]. Em média, durante a aula de step atlético as participantes passam mais 10 minutos em regime vigoroso do que na aula de step coreografado. Esta diferença deve-se essencialmente ao facto do intervalo de tempo em intensidades muito leves e leves ser superior na aula de step coreografado. Estes resultados podem ser justificados pelo facto da rotina da aula de step coreografado ser caraterizada por passos de dança, alguns mais complexos e com maior exigência de coordenação, o que leva a uma maior dificuldade na aquisição de alguns passos por parte dos praticantes. Por outro lado, a aula de step atlético caracteriza-se por ter uma coreografia com passos mais simples e atléticos e com maior repetição dos mesmos, o que permite às participantes interiorizarem mais rapidamente a sequência das ações motoras.

De acordo com os resultados apresentados, o parâmetro fisiológico selecionado (\%FCmáx ou \%FCreserva), afeta a quantificação do tempo passado em cada intervalo de intensidade, em particular na modalidade de step atlético. Segundo a ACSM (2011), embora possam ser utilizados diversos métodos para estimar a intensidade relativa do exercício cardiorrespiratório, não é possível assumir que um determinado método de estimativa é necessariamente equivalente à estimativa derivada de um outro método. É portanto prudente manter em mente que a relação entre os vários parâmetros de avaliação dependem do modo de teste e da intensidade do exercício, entre outros (ACSM, 2011). No entanto, a literatura tem indicado a \%FCreserva como um bom indicador para a população considerada sedentária ou ativa não-atleta (Swain, 2000) the use of net VO2 rather than gross VO2 for the calculation of caloric expenditure during exercise, and a modification of the American College of Sports Medicine (ACSM.

Embora este tipo de modalidades permita à população em geral cumprir as recomendações de atividade física necessárias à melhoria da saúde e bem-estar da mesma, os estudos neste âmbito são limitados. A manipulação de parâmetros como a cadência musical ou seleção de ações motoras, pode promover diferentes respostas agudas fisiológicas (Martinovic \& Novaes, 2002). Por exemplo, Zaletel, Furjan-Mandié, e Zagorc (2009) efetuaram um estudo onde se compararam os valores de FC ao longo de uma sessão de step de 30 minutos, utilizando uma coreografia 
padronizada, em três diferentes alturas de plataforma do step e verificaram que o valor médio da FC durante exercício aumentou de forma significativa com um aumento de $10 \mathrm{~cm}$ à altura da plataforma.

A influência da manipulação de outras variáveis subjacentes ao planeamento das aulas de step na resposta fisiológica dos praticantes não tem sido devidamente explorada. Num estudo realizado por Grossl et. al (2012), cujo objetivo foi determinar o perfil fisiológico de uma aula de Body Stepß (duração de 60 min, com plataforma de $15 \mathrm{~cm}$ ), verificaram uma FC média da aula de 147 bpm. No presente estudo, verificámos um valor FC média mais elevada [aula de step coreografado 157 bpm (79\% FCmáx); aula de step atlético 158 bpm (79,5\% FCmáx)]. Martinovic e Novaes (2002), ao analisarem a FC durante uma sequência de exercícios de step training durante 20 min, com ritmo musical de 132 bpm, a diferentes alturas da plataforma $(15 \mathrm{~cm}$ e $20 \mathrm{~cm})$, encontraram valores médios da FC de 138 bpm para a plataforma de 15 cm e 152 bpm para a plataforma de $20 \mathrm{~cm}$ (com diferença significativa entre eles). Estes valores corresponderam a $74 \%$ e $81 \%$ (plataformas de $15 \mathrm{~cm}$ e $20 \mathrm{~cm}$, respetivamente) da FCmáx. O presente estudo foi realizado em plataformas de $15 \mathrm{~cm}$, com uma cadência musical de 132 bpm, tendose observado valores superiores aos indicados por Martinovic e Novaes (2002). Esta disparidade poderá ser explicada em parte pelas diferenças na duração das avaliações de ambos os estudos (20 min vs. 45 min, respetivamente). Por outro lado Luettgen, Foster, Doberstein, Mikat e Porcari (2012), na realização de um estudo para determinar a intensidade e dispêndio energético durante uma aula de Zumba®, verificaram uma FC média de 154 bpm e uma \%FCmáx de 79\%. A intensidade média do esforço (medida através da \% FCmáx) observada por estes autores é semelhante ao verificado no presente estudo em ambas as vertentes da aula de step. Embora a modalidade Zumba® não recorra a plataformas, o tipo de metodologia utilizada induz tipicamente intensidades vigorosas (Domene, Moir, Pummell, \& Easton, 2016). No entanto, tal como já referido a análise dos valores médios não refletem o tempo total passado em cada intervalo de intensidade, podendo haver diferenças entre ambas as modalidades.

Este estudo procurou analisar o tempo total despendido em cada intervalo de intensidade, mostrando assim diferenças entre vertentes de step que não são descortinadas apenas pela análise dos valores médios das 
variáveis fisiológicas analisadas (\% FCreserva e \%FCmáx) ao longo de toda a aula. Contudo o presente estudo apresenta algumas limitações que importa referir. A principal limitação centra-se na dimensão e constituição da amostra. $\bigcirc$ grupo foi constituído por um número reduzido de jovens mulheres, o que pode limitar a extrapolação dos resultados para a população em geral. Cada vertente da aula de step foi apenas avaliada através de uma única sessão, sendo as avaliações mais vulneráveis a um efeito casualístico. Assim, e no sentido de melhor conhecer o impacto fisiológico das várias vertentes das modalidades de fitness, é aconselhável que estudos futuros envolvam amostras de maior dimensão e com diferentes características (género e grupos etários), implementando várias sessões de avaliação de forma randomizada.

\section{CONCLUSÃO}

No presente estudo observou-se que a média das variáveis fisiológicas (\% FCmáx e \%FCreserva), indica que ambas as vertentes induzem uma intensidade de esforço considerada vigorosa. Contudo uma análise por intervalos de tempo, em função das intensidades de esforço, mostrou que é na vertente step atlético que as participantes passam mais tempo em intensidade de esforço vigorosa (em média, 29 dos 45 minutos de duração da parte fundamental da aula). De acordo com estes resultados, a participação em 3 sessões semanais neste tipo de aula de step permitirá alcançar as recomendações do ACSM (2011) (75 minutos semanais de atividade física, de intensidade vigorosa). Por outro lado, a aula de step coreografado permitiu às participantes passar apenas 21 minutos em intensidade vigorosa. Nesta vertente verificou-se também que as participantes passaram mais tempo em intensidades muito leve e leve. Estes resultados indicam-nos que a observação do valor médio global de variáveis fisiológicas tais como a \%FCmáx ou \%FCreserva, não é um indicador preciso, devendo ser feita uma análise do tempo despendido em cada nível de intensidade ao longo de toda a aula. Só desta foram será possível verificar, com maior efetividade, o cumprimento das recomendações da ACSM no que diz respeito à melhoria e manutenção da aptidão cardiovascular (intensidade e duração do estímulo). 


\section{BIBLIOGRAFIA}

Acsm (2011). Quantity and quality of exercise for developing and maintaining cardiorespiratory, musculoskeletal, and neuromotor fitness in apparently healthy adults: guidance for prescribing exercise. Medicine \& science in sport \& exercise, 43(7), $1334-1359$.

Arslan, f. (201 1). The effects of an eight-week step-aerobic dance exercise programme on body composition parameters in middle-aged sedentary obese women. International sport med journal, 12(4), 160-168.

Borg, g. A. V. (1982). Psychophysical bases of perceived exertion. Medicine and science in sports and exercise, 14(5), 377-381.

De angelis, m., vinciguerra, g., gasbarri, a., \& pacitti, c. (1998). Oxygen uptake, heart rate and blood lactate concentration during a normal training session of an aerobic dance class. European journal of applied physiology and occupational physiology, 78(2), 121-127.

Desimone, g. (2012): acsm's resources for the group exercise instructor. Philadelphia, united states of america, lippincott williams \& wilkins.

Domene, p., moir, h., pummell, e., \& easton, c. (2016). Salsa dance and zumba fitness: acute responses during community-based classes. Journal of sport and health science 5 (2016) 190-196.

Fox, s.m., naughton, i.p. \& haskell, w.l. (1971). Physical activity and the prevention of coronary heart disease. Annals of clinical research, 3, 404-432.

Grier, t. D., Iloyd, I. K., walker, i. L., murray, t. D. (2002). Metabolic cost of aerobic dance bench stepping at varying cadences and bench heights. Journal of strength and conditioning research, 16 (2), 242-249.

Grossl, t., pires, c., silva, r. C. R., rosa, f., lucas, r., guglielmo, I. (2012). Perfil fisiológico de uma aula de body step. Rev. Educ. Fís/uem, 23(1), 87-96.

Jaywant, p.j. (20005): effect of aerobic dance on the body fat distribution and cardiovascular endurance in middle aged women. J. Exercise science and physiotherapy, 9 (1), 6-10.

Karvonen, m.j., kentala, k., \& musta, o. (1957). The effects of training heart rate: a longitudinal study. Annals of medicine and experimental biology, 35, 307-315. La torre, a., impellizzeri, f. M., rampinni, e., casanova, f., alberti, f., \& marcora, s. M. (2005). Cardiovascular responses to aerobic step dance sessions with and without appendicular overload. Journal of sports medicine and physical fitness, 45(3), 264- 269.

Laukkanen, r. M., kalaja, m. K., kalaja, s. P., holmala, e. B., paavolainen, I. M., tummavuori, m., ... rosko, h. K. (2001). Heart rate during aerobics classes in women with different previous experience of aerobics. European journal of applied physiology, 84(1-2), 64-68. 


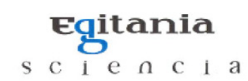

Luettgen, m., foster, c., doberstein, s., mikat, r., porcari, i. (2012). Zumba: is the "fitness-party" a good workout? Journal of sports science and medicine, 11 , 357-358.

Martinovic, n. \& novaes, i. (2002). Respostas cardiovasculares e metabólica do step training em diferentes alturas de plataforma. Atividade física e saúde, 7 (2), 5-11.

Reiner, m., niermann, c., jekauc, d., \& woll, a. (2013). Long-term health benefits of physical activity--a systematic review of longitudinal studies. Bmc public health, 13(1), 813.

Swain, d. P. (2000). Energy cost calculations for exercise prescription: an update. Sports medicine (auckland, n.z.), 30(1), 17-22.

Zaletel, p., furjan- mandié, g., zagorc, m. (2009). Differences in heart rate and lactate levels at three different workloads in step aerobics. Kinesiology, 41, 97 104.

Zar, i (2010): biostatistical analysis. 5th ed. New jersey, united states of america. Pearson printice hall. 